\title{
Identification des triacylglycérols en HPLC. Comment se passer du couplage HPLC-SM? Dans quel cas la chromatographie est-elle encore indispensable?
}

\author{
Arnaud W. G. Tamba Sompila ${ }^{1,2}$, Marie Geneviève Maloumbi ${ }^{1, \star}$, Jean Bleton ${ }^{2}$, Alain Tchapla ${ }^{2}$ \\ et Sylvie Héron ${ }^{2}$ \\ ${ }^{1}$ Laboratoire de Valorisation des Agro-Ressources (LVAR), École Nationale Supérieure Polytechnique (ENSP), BP 69, Brazzaville, Congo \\ 2 Université Paris Sud, Groupe de chimie analytique de Paris-Sud, EA 4041, LETIAM, IUT d'Orsay Plateau de Moulon, 91400 Orsay, France
}

Reçu le 12 mars 2014 - Accepté le 28 mai 2014

\begin{abstract}
Résumé - Les huiles et graisses sont principalement constituées de triacylglycérols. Ces derniers diffèrent par leur nombre total de carbone, la répartition des acides gras sur les trois sommets de la tête glycéryle et la configuration linéaire, ramifiée ou cyclique des chaînes grasses. Ils diffèrent aussi par leur nombre total de doubles liaisons, leur répartition sur les chaînes grasses, et au sein d'une chaîne grasse par leur position et leur configuration. Cela conduit à des mélanges complexes comprenant un grand nombre de congénères qu'il est classique d'analyser par chromatographie en phase liquide. Dans cet article, nous montrons qu'indépendamment du détecteur utilisé (DEDL, Corona, SM) la seule utilisation des lois de rétention $\ln \alpha=f(\mathrm{NC}), \ln \alpha=f(\mathrm{NDL})$ et $\ln \alpha=f(\mathrm{NP})$ permet d'identifier les triacylglycérols d'huiles d'Afrique équatoriale. La démarche proposée ne nécessite pas de couplage spécifique avec la spectrométrie de masse, un système HPLC de base suffit. À titre d'exemple nous avons choisi l'huile de Ricinodendron heudelotii qui contient majoritairement des acides gras polyinsaturés en C18:3 isomères de position et de configuration.
\end{abstract}

\begin{abstract}
Mots clés : Triacylglycérols / HPLC / lois de rétentions chromatographiques / Ricinodendron heudelotii
Abstract - Identification of triacylglycerols in HPLC. Can we do without HPLC-MS? In which case the chromatography is it still necessary? Oils and fats are mainly composed of triacylglycerols. These differ in their total number of carbon atoms, the distribution of fatty acids on the three chains of the glycerol, and the configuration of fatty chains, linear, branched or cyclic. They also differ in the total number of double bonds, their position and configuration. This leads to complex mixtures with many congeners widely analyzed by conventional liquid chromatography. In this paper we show that whatever the detector (ELSD, Corona, MS) using only retention laws $\ln \alpha v s$. NC, $\ln \alpha v s$. NDL and $\ln \alpha v s$. NP allows us the identification of triacylglycerols in Equatorial Africa oils. The proposed approach does not require specific coupling with mass spectrometry. As example, we chose Ricinodendron heudelotii oil which mainly contains polyunsaturated positional isomers and configuration fatty acids C18:3.
\end{abstract}

Keywords: Triacylglycerols / HPLC / retention laws / Ricinodendron heudelotii

\section{Introduction}

Les triacylglycérols (TAG), principaux constituants des corps gras, ont une structure simple : ce sont des triesters glycériques d'acides gras (AG). D'un corps gras à un autre ces AG peuvent différer selon leur nombre de carbone (NC), leur configuration linéaire, ramifiée ou cyclique, mais aussi selon le nombre (NDL), la position et la configuration cistrans des doubles liaisons présentes dans la chaîne grasse, ainsi que par leurs positions sur le squelette glycerol (isomères de position, régioisomères, énantiomères). Les TAG des corps gras sont constitués par une très grande diversité d'AG présents en proportions diverses et forment des mélanges naturels complexes à très grand nombre de congénères (Christie, 2003).

\footnotetext{
^ Correspondance : mgmaloumbi@yahoo.fr
}

À l'heure actuelle, une des techniques les plus performantes pour la caractérisation de la composition en TAG des corps gras est la chromatographie liquide (HPLC), car elle permet la séparation d'un grand nombre d'isomères. Deux chromatographies complémentaires sont couramment utilisées : d'une part la chromatographie liquide à polarité inversée de phases non aqueuse (NARP-LC), d'autre part la chromatographie d'argentation (Ag-LC) (Buchgraber et al., 2004; Christie, 2003).

La NARP-LC sépare principalement les différents congénères selon leur nombre de carbone total et pour un même $\mathrm{NC}$ selon le nombre de doubles liaisons (NDL), tandis que l'Ag-LC sépare principalement selon le nombre de doubles liaisons (NDL) (Nikolova-Damyanova, 2003). Cela conduit à une bonne orthogonalité entre ces deux méthodes et à la mise en place ces derniers temps de LC-2 dimensions en 
ligne permettant la caractérisation de mélanges très complexes (Dugo et al., 2004; François et Sandra, 2009; Hu et al., 2013; Yang et al., 2012).

Du point de vue de la détection, l'UV à faible longueur d'onde (205 ou $210 \mathrm{~nm}$ ), le détecteur évaporatif à diffusion de lumière (DEDL) et plus récemment le détecteur à décharge Corona sont couramment employés (Christie, 2003). Sachant qu'il existe très peu de TAG standards mixtes disponibles commercialement, le couplage chromatographie-spectrométrie de masse $(\mathrm{SM})$ ou $\left(\mathrm{SM}^{n}\right)$ pour résoudre l'identification des différents congénères est devenu très courant (Gómez-Ariza et al., 2006; Laakso et Voutilainen, 1996; Schuyl et al., 1998).

Si l'on ne s'intéresse pas à la séparation des TAG régioisomères ni aux TAG énantiomères et que l'on n'a pas d'information extérieure, l'utilisation des couplages HPLC-APCIMS (Lísa, Holčapek, 2008; Modello et al., 2005) ou bien du couplage HPLC-ESI-MS ${ }^{n}$ (Hsu et Turk, 1999; Zeng 2010) permet les identifications de TAG constitués de résidus saturés sans difficulté ou bien de TAG constitués de résidus insaturés mais sans localisation des doubles liaisons.

Dans le cas où on a l'information de la composition en AG, différents articles montrent la nécessité d'utiliser la complémentarité des informations déduites de la chromatographie et de la $\mathrm{SM}$ ou $\mathrm{SM}^{n}$ pour élucider la composition en TAG isomères de mélanges complexes (Achéampong et al., 2011; Lísa et Holčapek, 2008; Lísa et al., 2011; Řezanka et al., 2012). Ceci est particulièrement crucial dans le cas de TAG dont les AG constitutifs sont isomères de position ou de configuration, la simple SM ne permettant pas d'identifier de tels isomères. Deux articles récents offrent cette éventualité. Soit il faut faire de l'APCI-SM en mode négatif, mais la méthode est peu sensible (Yang et al., 2011), soit il faut faire de la dissociation dirigée radicalaire (RDD-MS ${ }^{n}$ ) avec une source modifiée pour obtenir des fragments caractéristiques de ces AG et pouvoir identifier en ligne les TAG correspondant (Pham et al., 2012). Cette dernière technique prometteuse n'a pas encore été décrite en couplage avec la HPLC; elle est encore du domaine de la R\&D et non pas de l'analyse de routine.

Dans ce travail, nous montrons qu'il n'est pas indispensable d'avoir un couplage avec la SM pour proposer une identification de TAG isomères lors d'analyses de routine en NARP-LC. L'utilisation des lois de rétention chromatographique permet de répondre au problème. Dans un premier temps, la démarche générale permettant cette identification est décrite, puis dans un second temps elle est mise en application sur une huile d'Afrique équatoriale possédant des TAG isomères de position en C18:3, Ricinodendron Heudelotii.

\section{Matériel et méthode}

\subsection{GC-MS}

L'huile a été transestérifiée et l'analyse des esters méthyliques obtenus effectuée.

L'appareillage utilisé est un chromatographe Thermo Scientific TRACE GS Ultra ${ }^{\mathrm{TM}}$ équipé d'un passeur d'échantillon Thermo Scientific AI 3000, couplé à un spectromètre de masse de type quadripolaire Thermo Scientific DSQ ${ }^{\mathrm{TM}}$ II et piloté par le logiciel Xcalibur (ThermoFisher, Les Ulis, France).
La colonne est une Supelco Simplicity Wax $10 \mathrm{~m} \times$ $0.25 \mathrm{~mm} \times 0.25 \mu \mathrm{m}$ (St Quentin Fallavier, France). L'injection se fait en mode split $1: 25$. Le volume d'échantillon est de $1 \mu 1$. La température de l'injecteur est $250^{\circ} \mathrm{C}$. Le gaz vecteur utilisé est l'hélium à un débit constant de $1,3 \mathrm{ml} / \mathrm{min}$. Le gradient de température va de 100 à $245^{\circ} \mathrm{C}$ avec une rampe de $10^{\circ} \mathrm{C} / \mathrm{min}$. L'ionisation électronique (EI) se fait à $70 \mathrm{eV}$. La température de la source est $250{ }^{\circ} \mathrm{C}$, celle de la ligne de transfert à $245^{\circ} \mathrm{C}$.

\subsection{NARP-LC}

L'acétonitrile, l'acétone et le chlorure de méthylène sont de qualité HPLC et achetés chez Carlo Erba (Rodano, Italy).

Les TAG homogènes standards tricaproïne (CoCoCo), tricapryline (CyCyCy), tricaprine (CCC), trilaurine (LaLaLa), trimyristine (MMM), tripalmitine (PPP), tristéarine (SSS), trioléine (OOO), trilinoléine (LLL), trilinolénine ( $\mathrm{LnLnLn)}$ et les esters méthyliques d'AG correspondant ont été achetés soit chez Cluzeau (Sainte Foy la Grande, France) soit chez Larodan (Malmö, Suède). L'huile de Ricinodendron Heudelotti a été récoltée en Côte d'Ivoire.

Certaines huiles ont aussi été utilisées comme standards. Leurs compositions étant décrites dans la littérature, leur injection a permis l'identification de certains TAG mixtes. Elles ont aussi été injectées en dopage avec l'huile Ricinodendron Heudelotti (Héron et al., 1995). Dans ce travail nous avons utilisé les huiles suivantes : l'huile de Calophyllum inophyllum et l'huile de pépin de cassis (Ribes nigrum) qui proviennent de l'Institut de recherche Pierre Fabre (Vigoulet, France). L'huile de grenade (Punica granatum) a été achetée chez AromaZone (Paris, France). L'huile de Tung encore appelée de bois de Chine (Aleurites fordi) provient de chez Laverdure (Paris, France), l'huile de pignon de pin (Pinus koraiensis) est un don de R.L. Wolff (ISTAB, Univ. Bordeaux I, Talence, France). Elles ont servis comme standards pour l'identification des TAG mixtes suivants (Héron, Tchapla, 1994) : $\operatorname{LnLn} \gamma \operatorname{Ln}, \operatorname{Ln} \gamma \operatorname{Ln} \gamma \operatorname{Ln}, \operatorname{LLnLn}, \operatorname{LLn} \gamma \operatorname{Ln}, \operatorname{L} \gamma \operatorname{Ln} \gamma \operatorname{Ln}, \operatorname{LLLn}$, LL $\gamma$ Ln, OL $\gamma$ Ln, PL $\gamma$ Ln, OLLn, PLLn, OLL, OOLn, PLL, POLn, OOL, SLL, POL, PPL, OOO, SOL, POO, PSL, PPO, SOO, SSL, PSO, SSO, $\alpha \mathrm{E} \alpha \mathrm{E} \alpha \mathrm{E}, \alpha \mathrm{E} \alpha \mathrm{E} \beta \mathrm{E}$ et PuPuPu.

Tous les échantillons ont été solubilisés dans un mélange $\mathrm{MeCN} / \mathrm{CH}_{2} \mathrm{Cl}_{2}$ 50/50.

Le système HPLC est constitué d'une pompe quaternaire Spectra System P1000XR (ThermoFisher, Les Ulis, France), d'une vanne d'injection Rhéodyne 7125 munie d'une boucle de $10 \mu \mathrm{L}$ (Rhéodyne, Cotati, CA), d'un four à effet Peltier Croco-cil (Cluzeau, Sainte Foy la Grande, France) maintenu à $25^{\circ} \mathrm{C}$ et d'un DEDL $75\left(P_{\text {air }}=3.5\right.$ bars, $\left.T=45^{\circ} \mathrm{C}\right)$ (Sedere, Alfortville, France). Le système d'acquisition Azur (v4.6) provient de chez Datalys (St Martin d'Hyères, France).

La colonne Kromasil 100-5C18, $250 \times 4.6$ mm $5 \mu \mathrm{m}$ provient de chez Akzo Nobel (Bohus, Suède). La phase mobile est $\mathrm{MeCN} /$ Acétone 30/70. Le débit de travail est de $1 \mathrm{ml} / \mathrm{min}$.

Pour chacun des TAG, le facteur de rétention $k$ a été déterminé à partir de la formule suivante : $k=\left(t_{r}-t_{0}\right) / t_{0}, t_{r}$ étant le temps de rétention du TAG et $t_{0}$, le temps mort déterminé par la méthode de la double pesée (Martin et al., 1988). 


\section{Résultats et discussion}

En NARP-LC, il est bien établi que l'ordre d'élution des TAG se fait selon le nombre de partition croissant (NP = NC-2.NDL) (Buchgraber et al., 2004). Avec les efficacités des colonnes actuelles, on sépare la plupart des TAG ayant le même NP, en suivant approximativement leur NDL (Lísa et Holčapek, 2008). Certains TAG isobares de même NP et NDL (isomères de position de doubles liaisons) ainsi que les TAG isobares isomères saturés ramifiés ou linéaires sont même séparés (Achéampong et al., 2011; Héron, Tchapla, 1994; Héron et al., 1995; Lísa et Holčapek, 2008). Bien que les chromatogrammes des TAG soient ordonnés en fonction de leur NP, il peut y avoir inversion d'ordre de sortie entre les derniers TAG d'un NP donné avec les premiers TAG du NP suivant (Achéampong et al., 2011).

Par conséquent, l'identification précise des TAG en utilisant la seule relation reliant NP à la rétention ne suffit pas, il faut aussi utiliser les lois reliant la rétention au NC et au NDL.

Le principe est basé sur une approche thermodynamique de la rétention chromatographique reliant la rétention à la structure de solutés homologues, tels les TAG. Les lois qui en résultent relient NP, NC ou NDL soit à leur $\ln k$ en analyse isocratique soit à leur temps de sortie réduit $t_{\mathrm{rG}}$ ' en gradient d'élution (Goiffon et al., 1981a, 1981b ; Podlaha, Töregard, 1982 ; Semporé, Bézard, 1986; Stołyhwo et al., 1985).

Dans le cas des TAG isologues (contenant au moins un AG saturé homologue, par exemple $\mathrm{Co}, \mathrm{Cy}, \mathrm{C}, \mathrm{La}, \mathrm{M}, \mathrm{P}, \mathrm{S})$ il a été montré que les relations $\ln k=f(\mathrm{NC})$ ou $t_{\mathrm{rG}}^{\prime}=f(\mathrm{NC})$ sont des droites aussi bien pour les TAG homogènes XXX que les TAG mixtes de structure XYY [CoYY-CyYY-CYY-LaYYMYY-PYY] et de structure XYZ [CoYZ-CyYZ-CYZ-LaYZMYZ-PYZ]. La représentation graphique de $\ln k=f(\mathrm{NC})$ pour ces trois séries de TAG conduit à l'obtention des trois droites parallèles quels que soient les résidus $\mathrm{Y}$ et $\mathrm{Z}$. (Tchapla et al., 1993). De manière plus générale il a été montré que, quelle que soit la structure saturée ou insaturée des AG X, Y et $\mathrm{Z}$ constituant des TAG différant par leur NC, les courbes $\ln k=f(\mathrm{NC})$ sont des droites parallèles pour les deux séries [XXX-XXY-XYY-YYY] et [XXZ-XYZ-YYZ].

Le nombre de partition NP, étant une grandeur dérivée de $\mathrm{NC}$, les mêmes lois de rétention peuvent alors être observées non plus en fonction de NC mais de NP. La représentation graphique $\ln k=f(\mathrm{NP})$ magnifie l'effet des DL. Elle permet de mieux visualiser la rétention de TAG de même NC possédant un NDL différent. Enfin elle transcrit l'allure du chromatogramme.

Finalement, en ne prenant en compte que le NDL des TAG de même NP ou de même NC, il a été montré que si l'on remplace un AG constitutif X par un AG Y de NDL différent, les courbes $\ln k=f(\mathrm{NDL})$ ou $t_{\mathrm{rG}}^{\prime}=f(\mathrm{NDL})$ sont elles aussi des droites parallèles pour les deux séries de TAG [XXX-XXYXYY-YYY] et [XXZ-XYZ-YYZ]. Ce dernier tracé permet une identification plus aisée et complémentaire à celle déduite des deux tracés précédents (ln $k$ ou $t_{\mathrm{rG}}^{\prime}$ en fonction de NP ou NC) dans la mesure où il provoque une extension d'échelle sur l'axe des abscisses et permet d'éclater sur cet axe des TAG qui se trouvent sur une même verticale dans les deux premiers tracés.

Pour décrire la démarche d'identification proposée, nous avons choisi parmi les huiles africaines l'huile de
Tableau 1. Composition en acides gras de l'huile de Ricinodendron heudelotii.

\begin{tabular}{ccccc}
\hline Nom de l'AG & Abréviation & $\mathrm{NC}$ & $\mathrm{NDL}$ & $\%$ aire \\
\hline Acide palmitique & $\mathrm{P}$ & 16 & 0 & 5.4 \\
Acide stéarique & $\mathrm{S}$ & 18 & 0 & 8 \\
Acide oléique & $\mathrm{O}$ & 18 & $1(9 \mathrm{c})$ & 11.2 \\
Acide linoléique & $\mathrm{L}$ & 18 & $2(9 \mathrm{c} 12 \mathrm{c})$ & 28.1 \\
Acide punicique & $\mathrm{Pu}$ & 18 & $3(9 \mathrm{c} 11 \mathrm{t} 13 \mathrm{c})$ & 1.3 \\
Acide $\alpha$ éléostéarique & $\alpha \mathrm{E}$ & 18 & $3(9 \mathrm{c} 11 \mathrm{t} 13 \mathrm{t})$ & 35 \\
Acide catalpique & $\mathrm{Ca}$ & 18 & $3(9 \mathrm{t} 11 \mathrm{t} 13 \mathrm{c})$ & 1.3 \\
Acide $\beta$ éléostéarique & $\beta \mathrm{E}$ & 18 & $3(9 \mathrm{t} 11 \mathrm{t} 13 \mathrm{t})$ & 9.7 \\
\hline
\end{tabular}

Ricinodendron heudelotii. Comme préconisé par différents auteurs (Goiffon et al., 1981b; Lísa et Holčapek, 2008; Stołyhwo et al., 1985), afin de minimiser les erreurs d'identification dues à de subtiles dérives des temps de rétention, nous avons pris OOO comme référence, travaillé en sélectivité ( $\alpha=k_{\mathrm{TAG}} / k_{\mathrm{OOO}}$ ) et utilisé les relations linéaires $\alpha$ en fonction de NC, NDL ou NP pour attribuer une structure de TAG à un pic chromatographique.

\subsection{Résultats préliminaires}

La première étape consiste à déterminer la composition en AG du corps gras par analyse CGC-SM des esters méthyliques obtenus après sa transestérification (Achéampong et al., 2011). L'identification des AG a été faite grâce à la banque de données de SM, par comparaison avec une injection des esters méthyliques d'AG standards purs ou en tenant compte des résultats de la littérature (Ucciani, 1995). La composition est reportée Tableau 1.

A partir de cette composition, un calcul statistique basé sur une distribution aléatoire des AG sur les trois carbones du glycérol a été effectué (Blank et Privett, 1966; Merritt et al., 1982). Il donne l'abondance probable des TAG observables par analyse d'un corps gras. Il est important de noter que la répartition naturelle ne suit pas systématiquement cette distribution au hasard ; un TAG dont le pourcentage est statistiquement très abondant peut en réalité être minoritaire ou absent. Par contre si le calcul attribue un faible pourcentage à un TAG, ce dernier ne peut pas être expérimentalement un TAG majoritaire ou très abondant (Achéampong et al., 2011; Zeitoun et al., 1991). Ce tableau préliminaire (non donné ici) sert donc de guide pour l'attribution d'un ou plusieurs TAG à un pic chromatographique en tenant compte de son abondance expérimentale (pourcentage en aire du pic en HPLC) qui sera comparée à l'abondance statistique calculée du TAG en tenant compte des limites décrites ci-dessus.

\subsection{Identification des TAG de I'huile Ricinodendron Heudelotii}

Cette huile a été prise en exemple car elle est majoritaire en AG polyinsaturés isomères qui diffèrent entre eux par la position des doubles liaisons (Kapseu et Tchiegang, 1995; Ucciani, 1995; Yeboah et al., 2011; Ghomdim Nzali et al., 2012, Aboubakar Dandjouma et al., 2013). Nous avons 
identifié plusieurs acides gras isomères en C18:3 (cf. Tab. 1) qui diffèrent par la configuration des doubles liaisons. Cette huile est majoritairement constituée d'acide $\alpha$ éléostéarique $(\mathrm{C} 18: 3(9 \mathrm{c} 11 \mathrm{t} 13 \mathrm{t}))$ tout en ayant des proportions non négligeables d'acide $\beta$ éléostéarique (C18:3 (9t11t13t)) auxquelles s'ajoutent l'acide punicique (C18:3 (9c11t13c)) et l'acide catalpique (C18:3 (9t11t13c)).

Une telle composition est en accord avec les résultats de la littérature : l'acide catalpique a déjà été décrit comme composant de cette huile (Ca : C18:3 (9t11t13c)) (PambouTchivounda et al., 1992). Etant donné les ordres de sortie observés en CGC, nous avons fait l'hypothèse que le quatrième isomère est l'acide punicique (Pu : C18:3 (9c11t13c)). Pour l'identifier nous avons alors analysé de l'huile de grenade (Punica granatum) dont la transestérification a conduit à un pourcentage en aire de $72 \%$ de $\mathrm{Pu}$ et $4,6 \%$ de $\alpha \mathrm{E}$, résultats conformes à la littérature (Ucciani, 1995).

Les TAG statistiquement les plus abondants correspondant à une telle composition primaire en $\mathrm{AG}$ étant constitués des résidus $\mathrm{P}, \mathrm{O}$ et $\mathrm{L}$ en plus des 4 résidus isomères en $\mathrm{C} 18: 3$, l'huile de Calophyllum inophyllum a été injectée dans les mêmes conditions afin de repérer la position des TAG mixtes « classiques » composés de $\mathrm{P}, \mathrm{S}, \mathrm{O}$ et $\mathrm{L}$ (voir matériel et méthode). Le tracé de $\ln \alpha=f(\mathrm{NDL})$ a été effectué en reportant dans un premier temps sur le diagramme les TAG «classiques » et les TAG standards homogènes correspondant (Fig. 1). Pour des raisons de lisibilité, tous les TAG n'y ont pas été indexés.

Les TAG majoritaires attendus étant constitués des résidus $\alpha \mathrm{E}$ et de $\beta \mathrm{E}$, le chromatogramme a été comparé à celui de l'huile d'Aleurites fordi (huile de Tung ou de bois de Chine). En effet, cette huile contient en majorité ces mêmes AG (Ucciani, 1995) mais dans des proportions différentes (73,1\% en $\alpha \mathrm{E}$ et 5,8\% en $\beta \mathrm{E}$ ). Cette comparaison ainsi que le mélange de ces 2 huiles (méthode d'identification par dopage préalablement décrite (Héron et al., 1995)) a permis d'identifier les 2 TAG $\alpha \mathrm{E} \alpha \mathrm{E} \alpha \mathrm{E}$ et $\alpha \mathrm{E} \alpha \mathrm{E} \beta \mathrm{E}$ qui ont le même NP mais des temps de rétention différents. Il n'est pas étonnant de voir que ces deux TAG isomères de position sont relativement bien séparés dans la mesure où d'autres TAG isomères de position du même type tel que $\operatorname{LnLn} \gamma \operatorname{Ln}$ et $\operatorname{LnLnLn}$ le sont aussi $(\gamma \mathrm{Ln}=$ C18:3 (6c9c12c), Ln = C18:3 (9c12c15c)) (Héron et al. 1995 ; Lisa, Holčapek 2008; Lisa et al. 2009), de même pour les TAG comprenant des résidus d'acides pinoléique $(\mathrm{Pi}=\mathrm{C} 18: 3$ (5c9c12c)) et linolénique (Achéampong et al., 2011).

L'étape suivante a consisté à déterminer les temps de rétention des TAG de type XXY et XYY contenant dans un premier temps les résidus $\mathrm{P}, \mathrm{O}, \mathrm{L}, \mathrm{S}$ et $\alpha \mathrm{E}$. En utilisant le graphe $\ln \alpha=f(\mathrm{NDL})$, les TAG $\mathrm{L} \alpha \mathrm{E} \alpha \mathrm{E}$ et $\mathrm{LL} \alpha \mathrm{E}$ ont été positionnés sur ce diagramme à partir de LLL et $\alpha \mathrm{E} \alpha \mathrm{E} \alpha \mathrm{E}$, (LLL$\mathrm{LL} \alpha \mathrm{E}-\mathrm{L} \alpha \mathrm{E} \alpha \mathrm{E}-\alpha \mathrm{E} \alpha \mathrm{E} \alpha \mathrm{E}$ formant une droite). Nous avons suivi le même raisonnement pour $\mathrm{P} \alpha \mathrm{E} \alpha \mathrm{E}, \mathrm{PP} \alpha \mathrm{E}, \mathrm{O} \alpha \mathrm{E} \alpha \mathrm{E}, \mathrm{OO} \alpha \mathrm{E}$, $\mathrm{S} \alpha \mathrm{E} \alpha \mathrm{E}$ et $\mathrm{SS} \alpha \mathrm{E}$.

Pour placer les TAG de type XYZ de NDL et NC différents, les droites de type XXY-XYZ-ZZY ont été utilisées. Puis la position de $X Y Z$ a été confirmée en traçant les deuX droites XXZ-XYZ-YYZ et XYY-XYZ-ZZX. Nous avons procédé ainsi pour positionner OL $\alpha \mathrm{E}, \mathrm{PL} \alpha \mathrm{E}, \mathrm{PO} \alpha \mathrm{E}, \mathrm{SL} \alpha \mathrm{E}$.

Nous nous sommes ensuite attachés à positionner les TAG composés de $\beta \mathrm{E}$. La connaissance de la rétention de $\alpha \mathrm{E} \alpha \mathrm{E} \alpha \mathrm{E}$

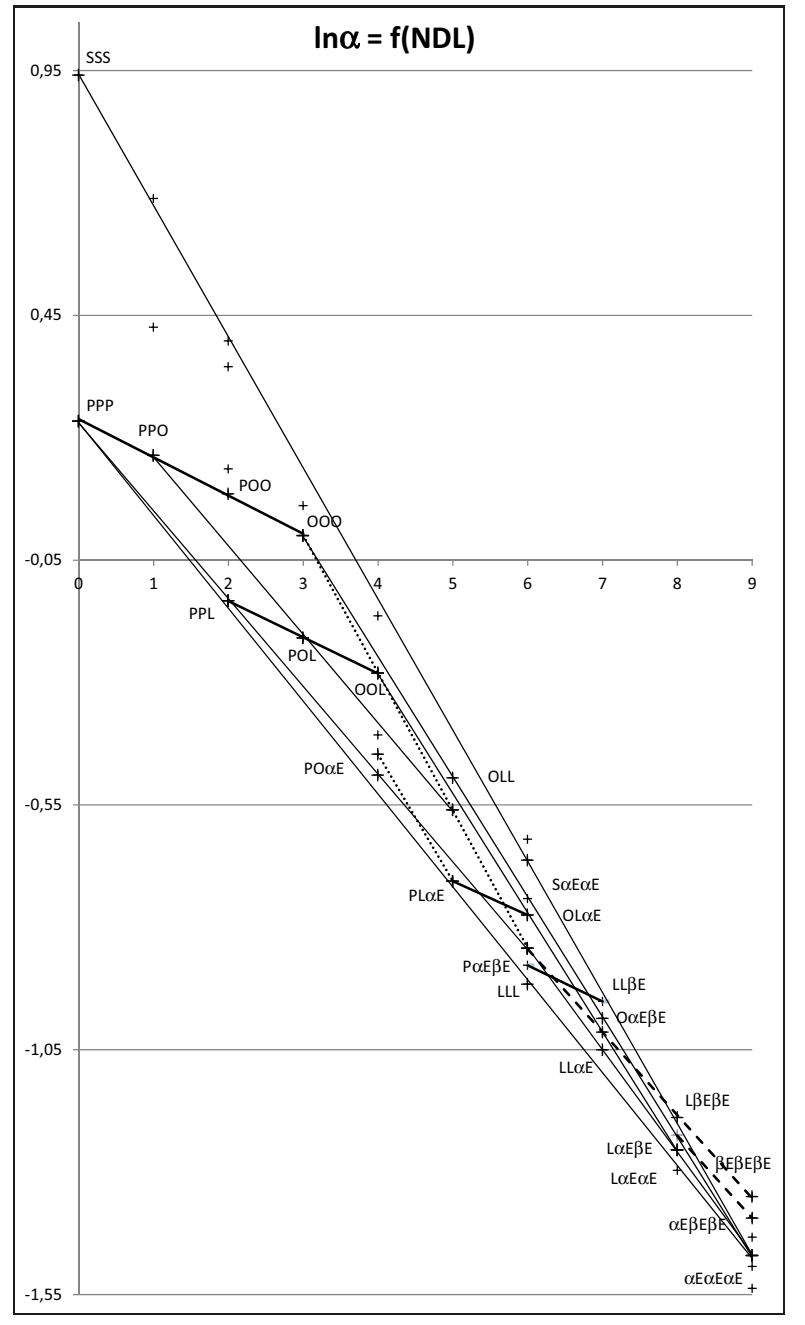

Fig. 1. Tracé de $\ln \alpha=f(\mathrm{NDL})$ pour l'identification des TAG de l'huile de Ricinodendron Heudelotii.

et $\alpha \mathrm{E} \alpha \mathrm{E} \beta \mathrm{E}$ conduit à la détermination de la sélectivité quand on remplace un résidu $\alpha \mathrm{E}$ par un résidu $\beta \mathrm{E}$. Cette sélectivité étant la même quels que soient les deux autres résidus AG présent sur les TAG considérés ( $\mathrm{XY} \alpha \mathrm{E}, \mathrm{XY} \beta \mathrm{E})$, il en a été déduit la position de $\alpha \mathrm{E} \beta \mathrm{E} \beta \mathrm{E}, \beta \mathrm{E} \beta \mathrm{E} \beta \mathrm{E}, \mathrm{LL} \beta \mathrm{E}, \mathrm{L} \beta \mathrm{E} \beta \mathrm{E}$ et $\mathrm{L} \alpha \mathrm{E} \beta \mathrm{E}$. Tous les TAG contenant du $\beta \mathrm{E}$ ont ainsi été placés, en vérifiant par ailleurs que la sélectivité entre $\mathrm{XY} \alpha \mathrm{E}$ et $\mathrm{XY} \beta \mathrm{E}$ soit bien constante, ce qui a permis une validation de la position de tous ces TAG.

En plus des deux AG majoritaires $\alpha \mathrm{E}$ et $\beta \mathrm{E}$, deux autres AG en C18:3 sont présents (Tab. 1). L'abondance statistique des TAG de l'huile de grenade conduit à ce que le TAG homogène PuPuPu soit le TAG majoritaire de cette huile. L'injection de cette huile nous a permis de repérer la position de ce TAG (il sort avant les TAG $\alpha \mathrm{E} \alpha \mathrm{E} \alpha \mathrm{E}$ et $\alpha \mathrm{E} \alpha \mathrm{E} \beta \mathrm{E}$ ) puis de déterminer celles de $\alpha \mathrm{E} \alpha \mathrm{EPu}$ et de $\alpha \mathrm{EPuPu}$. Cela nous a conduit à conclure que le premier pic des TAG constitués des seuls résidus $\mathrm{C} 18: 3$ est le TAG $\alpha \mathrm{E} \alpha \mathrm{EPu}$. En ce qui concerne le TAG analogue attendu contenant du $\mathrm{Ca}: \alpha \mathrm{E} \alpha \mathrm{ECa}$, tous les pics de NP = 36 étant indexés, nous pensons qu'il est masqué dans le doublet $\alpha \mathrm{E} \alpha \mathrm{E} \alpha \mathrm{E}$ et $\alpha \mathrm{E} \alpha \mathrm{E} \beta \mathrm{E}$. Ceci serait en accord avec les lois de rétention chromatographiques en RPLC qui 


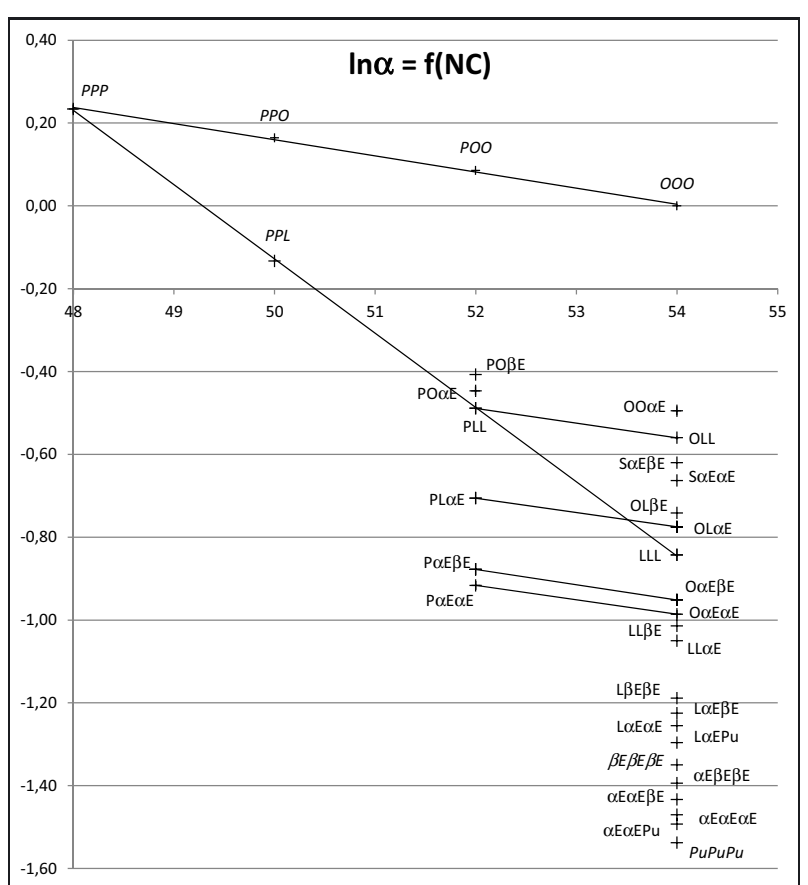

Fig. 2. Tracé de $\ln \alpha=f(\mathrm{NC})$ pour l'identification des TAG de l'huile de Ricinodendron Heudelotii.

montrent que les isomères cis sortent avant les isomères trans correspondant (Stołyhwo et al., 1985).

Le seul diagramme $\ln \alpha=f(\mathrm{NDL})$ permet d'identifier tous les TAG de l'huile de Ricinodendron Heudelotii. Vu que la plupart des TAG constituant cette huile possède un nombre de carbone identique, le tracé $\ln \alpha=f(\mathrm{NC})$ donné Figure 2 n'aide pas à l'identification, si ce n'est qu'à la vérification de la position des TAG de type PXY-OXY qui doivent se trouver sur des droites parallèles. Le tracé $\ln \alpha=f(\mathrm{NP})$ donné Figure 3 permet la confirmation des positions des TAG, déterminées sur les autres diagrammes dans la mesure où l'on doit retrouver des séries de parallèles. Les TAG étant positionnés dans l'ordre de NP, ce tracé permet d'avoir une représentation de l'ordre de sortie que l'on retrouve sur le chromatogramme. Il permet de constater que pour cette huile il n'y a pas de recouvrement de TAG ayant des NP successifs.

Au final, la comparaison des temps de rétention calculés de tous ces TAG, ajoutée à l'utilisation de leur pourcentage statistique calculé conduisent à l'identification donnée Tableau 2 et au chromatogramme Figure 4.

Les conditions de NARP utilisées permettant la séparation de TAG comportant des AG isomères de position, nous avons pu proposer une indexation des TAG de l'huile de Ricinodendron Heudelotii basée sur des seules informations chromatographiques. A notre connaissance aucune indexation complète des TAG de cette huile n'a été proposée à ce jour. En effet, la seule composition en TAG que nous ayons trouvée dans la littérature regroupe tous les TAG contenant des AG $\mathrm{C} 18: 3$, sans distinction des isomères de position (PambouTchivounda et al., 1992). Il est à noter qu'à l'heure actuelle le couplage en routine avec la SM ne permet pas de résoudre ce problème dans la mesure où les fragments obtenus ne permettent pas d'identifier les positions des doubles liaisons. Dans

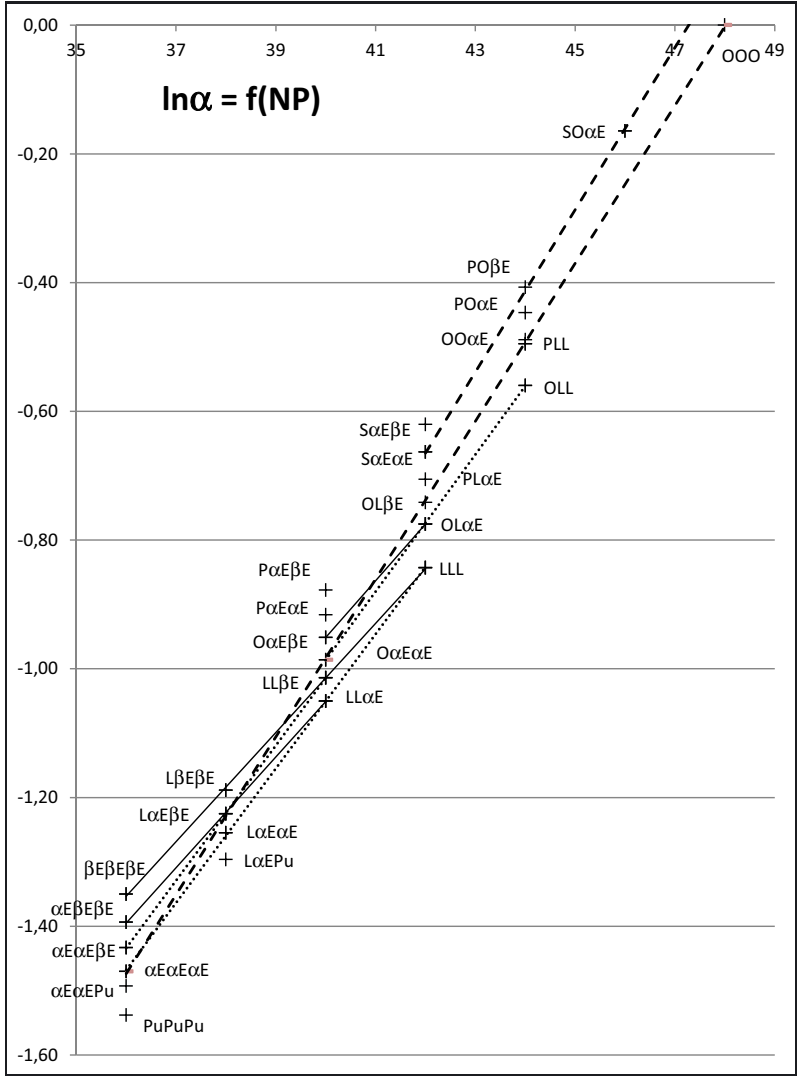

Fig. 3. Tracé de $\ln \alpha=f(\mathrm{NP})$ pour l'identification des TAG de l'huile de Ricinodendron Heudelotii.

ce cas précis, seuls les calculs utilisant les lois chromatographiques et éventuellement les dopages avec d'autres huiles judicieusement choisies peuvent le permettre.

\section{Conclusion}

De nos jours, pour toute identification structurale, le couplage HPLC-SM ou encore mieux le couplage HPLC-SM ${ }^{n}$ apparaît indispensable. Cependant, bien que de plus en plus répandu, c'est un matériel onéreux qui demande un entretien régulier et des conditions adaptées (salle climatisée et blanche, coûts de fonctionnement et d'entretien, pièces détachées...). Son implantation dans les pays ou les centres fortement industrialisés se démocratise de plus en plus, tandis que c'est beaucoup plus compliqué dans les pays faiblement industrialisés tels que les pays africains où les laboratoires possèdent un parc de matériel beaucoup moins sophistiqué.

En parallèle, vu la richesse et la diversité de produits végétaux dans les pays africains, la caractérisation de ces produits naturels est indispensable et doit s'adapter aux contingences locales.

Dans le cas particulier des huiles et corps gras végétaux et animaux contenant principalement des triacylglycérols, une fois la composition en AG constitutifs connue, nous avons montré dans cet article qu'il est possible de se passer de la SM pour l'identification des TAG en chromatographie en phase liquide. Ces composés constituant des séries homologues et 
Tableau 2. Composition en triacylglycerols de l'huile de Ricinodendron heudelotii. Conditions expérimentales données dans la partie expérimentale.

\begin{tabular}{|c|c|c|}
\hline $\begin{array}{c}\text { Temps de } \\
\text { rétention ( } \mathrm{min} \text { ) }\end{array}$ & $\mathrm{N}^{\circ}$ pic & Indexation \\
\hline 9.31 & 1 & $\alpha \mathrm{E} \alpha \mathrm{EPu}$ \\
\hline 9.57 & 2 & $\alpha \mathrm{E} \alpha \mathrm{E} \alpha \mathrm{E}$ \\
\hline 9.84 & 3 & $\alpha \mathrm{E} \alpha \mathrm{E} \beta \mathrm{E}$ \\
\hline 10.14 & 4 & $\alpha \mathrm{E} \beta \mathrm{E} \beta \mathrm{E}$ \\
\hline 10.58 & 5 & NI \\
\hline 10.93 & 6 & $\mathrm{~L} \alpha \mathrm{EPu}$ \\
\hline 11.29 & 7 & $\mathrm{~L} \alpha \mathrm{E} \alpha \mathrm{E}$ \\
\hline 11.56 & 8 & $\mathrm{~L} \alpha \mathrm{E} \beta \mathrm{E}$ \\
\hline 11.90 & 9 & $\mathrm{~L} \beta \mathrm{E} \beta \mathrm{E}$ \\
\hline 13.31 & 10 & $\operatorname{LL} \alpha \mathrm{E}$ \\
\hline 13.71 & 11 & $\operatorname{LL} \beta \mathrm{E}$ \\
\hline 14.03 & 12 & $\mathrm{O} \alpha \mathrm{E} \alpha \mathrm{E}$ \\
\hline 14.44 & 13 & $\mathrm{O} \alpha \mathrm{E} \beta \mathrm{E}$ \\
\hline 14.87 & 14 & $\mathrm{P} \alpha \mathrm{E} \alpha \mathrm{E}$ \\
\hline 15.36 & 15 & $\mathrm{P} \alpha \mathrm{E} \beta \mathrm{E}$ \\
\hline 15.89 & 16 & LLL \\
\hline 16.76 & 17 & $\mathrm{OL} \alpha \mathrm{E}$ \\
\hline 17.25 & 18 & $\mathrm{OL} \beta \mathrm{E}$ \\
\hline 17.79 & 19 & PL $\alpha \mathrm{E}$ \\
\hline 18.46 & 20 & $\mathrm{~S} \alpha \mathrm{E} \alpha \mathrm{E}$ \\
\hline 19.16 & 21 & $\mathrm{~S} \alpha \mathrm{E} \beta \mathrm{E}$ \\
\hline 20.06 & 22 & OLL \\
\hline 21.19 & 23 & PLL \\
\hline 21.40 & 24 & $\mathrm{OO} \alpha \mathrm{E}$ \\
\hline 22.34 & 25 & $\mathrm{PO} \alpha \mathrm{E}+\mathrm{SL} \alpha \mathrm{E}$ \\
\hline 23.14 & 26 & $\mathrm{PO} \beta \mathrm{E}+\mathrm{SL} \beta \mathrm{E}$ \\
\hline 28.84 & 27 & $\mathrm{SO} \alpha \mathrm{E}$ \\
\hline
\end{tabular}

$\mathrm{NI}$ : non identifié.

pouvant être isomères les uns des autres, nous avons utilisé des lois de rétention chromatographiques complémentaires nécessitant uniquement les informations chromatographiques de composés témoins, sans détecteur d'identification structurale. La méthode proposée nécessite seulement de posséder des composés témoins. Ceux-ci peuvent être commercialisés (TAG homogènes) ou bien des corps gras de référence dont la composition a été décrite sans ambiguitté dans la littérature. Dans ce cas, un laboratoire peut les avoir comme témoins dans une chimiothèque de réserve permettant de couvrir l'ensemble de la gamme des TAG mixtes nécessaires à une identification d'une huile dont la composition inconnue à ce jour doit être effectuée.

La méthode ne dépend pas du type de TAG (les AG le constituant pouvant être saturés ou polyinsaturés) ni du mode d'élution (isocratique ou gradient) (résultats non montrés ici).

Enfin, cette démarche s'avère même indispensable lorsqu'il s'agit d'identifier des TAG isomères de même NC, même NDL mais qui ne diffèrent que par la position ou la configuration des doubles liaisons au sein de la chaîne grasse, cas extrême où la HPLC-SM de routine est encore tenue en échec à ce jour.

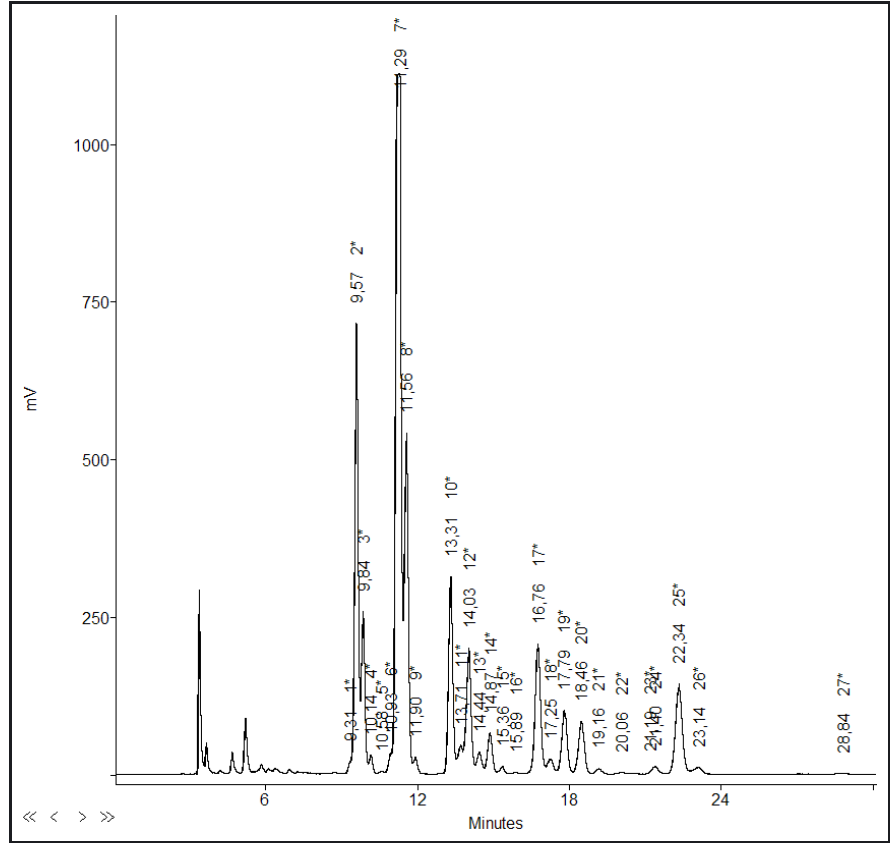

Fig. 4. Chromatogramme de l'huile de Ricinodendron Heudelotii. Conditions expérimentales données dans la partie expérimentale.

\section{Références}

Aboubakar Dandjouma AK, Tchiegang C, Bertrand NG. 2013. Quality of Ricinodendron heudelotii (Bail.) pierre ex pax seeds oil as affected by heating. Int. J. Eng. Res. Sci. Tech. 2: 94-100.

Achéampong A, Lévêque N, Tchapla A, Héron S. 2011. Simple complementary liquid chromatography and mass spectrometry approaches for the characterization of triacylglycerols in Pinus koraiensis seed oil. J. Chromatogr. A 1218: 5087-5100.

Blank ML, Privett OS. 1966. Evaluation of mathematic al distribution methods for the determination of triglyceride composition. Lipids 1: 27-30.

Buchgraber M, Ulberth F, Emons H, Anklam E. 2004. Triacylglycerol profiling by using chromatographic techniques. Eur. J. Lipid Sci. Technol. 106: 621-648.

Christie WW. 2003. Lipid analysis. Isolation, separation, identification and structural analysis of lipids. Bridgwater: The Oily Press.

Dubois V, Breton S, Linder M, Fanni J, Parmentier M. 2008. Proposition de classement des sources végétales d'acides gras en fonction de leur profil nutritionnel. OCL 15: 56-75.

Dugo P, Favoino O, Tranchida P.Q, Dugo G, Mondello L. 2004. Offline coupling of non-aqueous reversed-phase and silver ion highperformance liquid chromatography-mass spectrometry for the characterization of rice oil triacylglycerol positional isomers. $J$. Chromatogr. A 1041: 135-142.

François I, Sandra P. 2009. Comprehensive supercritical fluid chromatography $\mathrm{x}$ reversed phase liquid chromatography for the analysis of the fatty acids in fish oil J. Chromatogr. A 1216: 40054012.

Foma M, Abdala T. 1985. Kernel oils of seven plant species of Zaire. J. Am. Oil Chem. Soc. 62: 910-911.

Ghomdim Nzali H, Tchiengang C, Mignolet E, Turu C, Larondelle Y, Meurens M. 2012 Study of bioconversion of conjugated linolenic acid of Ricinodendron heudelotii (Bail.). Seed in male rats conjugated linoleic acid (CLA) using UV-vis spectrometry and gas chromatography. Asian J. biochem. 7: 194-205. 
Goiffon JP, Reminiac C, Olle M. 1981. High Performance liquid chromatography for fat triglyceride analysis. I. Search for the best operating conditions for soya-bean oil. Rev. Fr. Corps Gras 28: $167-170$.

Goiffon JP, Reminiac C, Furon D. 1981. High Performance liquid chromatography for fat triglyceride analysis. II. Retention indices of triglycerides. Rev. Fr. Corps Gras 28: 199-207.

Gómez-Ariza JL, Arias-Borrego A, García-Barrera T. 2006. Use of flow injection atmospheric pressure photoionization quadrupole time-of-flight mass spectrometry for fast olive oil fingerprinting. Rapid Commun. Mass Spectrom. 20: 1181-1186.

Héron S, Tchapla A. 1994. Finger prints of triacylglycerols from oils and fats by HPLC isocratic elution and evaporative light scattering detection. Alfortville : ELSD Sedex 45, Sedere.

Héron S, Lesellier E, Tchapla A. 1995. Analysis of triacylglycerols of borage oil by RPLC identification by coinjection. J. Liq. Chrom. 18: 599-611.

Hsu FF, Turk J. 1999. Structural characterization of triacylglycerols as lithiated adducts by electrospray ionisation mass spectrometry using low-energy collisionally activated dissociation on a triple stage quadrupole instrument. J. Am. Soc. Mass Spectrom. 10: 587-599.

Hu J, Wei F, Dong XY, LV X, Jiang ML, Li GM, Chen H. 2013. Characterization and quantification of triacylglycerols in peanut oil by off-line comprehensive two-dimensional liquid chromatography coupled with atmospheric pressure chemical ionization mass spectrometry. J. Sep. Sci. 36: 288-300.

Kapseu C, Tchiegang C. 1995. Chemical properties of Ricinodendron heudelotii (Bail.) seed oil. J. Food Lipids 2: 87-98.

Kapseu C, 2009 Production, analyse et applications des huiles végétales en Afrique. OCL 16: 215-229.

Laakso P, Voutilainen P. 1996. Analysis of triacylglycerols by silver-ion high performance liquid chromatography-atmospheric pressure chemical ionization mass spectrometry. Lipids 31: 1311-1322.

Lísa M, Holčapek M. 2008. Triacylglycerols profiling in plant oils important in food industry, dietetics and cosmetics using high-performance liquid chromatography-atmospheric pressure chemical ionization mass spectrometry. J. Chromatogr. A 1198-1199 : 115-130.

Lísa M, Holčapek M, Sovová H. 2009. Comparison of various types of stationary phases in non-aqueous reversed-phase highperformance liquid chromatography-mass spectrometry of glycerolipids in blackcurrant oil and its enzymatic hydrolysis mixture. J. Chromatogr. A 1216: 8371-8378.

Lísa M, Netušilová K, Franek L, Dvoráková H, Vrkoslav V, Holčapek M. 2011. Characterization of fatty acid and triacylglycerol composition in animal fats using silver-ion and non-aqueous reversedphase high-performance liquid chromatography / mass spectrometry and gas chromatography / flame ionization detection. $J$. Chromatogr. A 1218: 7499-7510.

Martin M, Thévenon G, Tchapla A. 1988. Comparison of retention mechanisms of homologous series and triglycerides in nonaqueous reversed-phase liquid chromatography J. Chromatogr. 452: $157-173$.

Merritt CJr, Vajdi M, Kayser SG, Halliday JW, Bazinet ML. 1982. Validation of computational methods for triglyceride composition of fats and oils by liquid chromatography and mass spectrometry. J. Am. Oil Chem. Soc. 59: 422-432.
Modello L, Tranchida P.Q, Stanek V, Jandera P, Dugo G, Dugo P. 2005. Silver-ion reversed-phase comprehensive two-dimensional liquid chromatography combined with mass spectrometric detection in lipidic food analysis J. Chromatogr. A 1086: 91-98.

Nikolova-Damyanova B. Lipid analysis by silver ion chromatography, In : Adlof RO, ed. Advances in Lipid Methodology-Five. Bridgwater (England) : The Oily Press, 2003.

Pambou-Tchivounda H, Koudogbo B, Pouet Y, Casadevall E. 1992. Composition en acide gras et en triglycérides des graines comestibles de cinq arbres de la forêt gabonaise. Rev. Fr. Corps Gras 39: 133-192.

Pham HT, Ly T, Trevitt AJ, Mitchell TW, Blanksby SJ. 2012. Differentiation of complex lipid isomers by radical-directed dissociation mass spectrometry. Anal. Chem. 84: 7525-7532.

Podlaha O, Töregard B. 1982. A system for identification of triglycerides in RP-HPLC chromatograms based on equivalent carbon numbers. J. High Resol. Chromatogr. 5: 553-558.

Řezanka T, Lukavský J, Siristova L, Sigler K. 2012. Regioisomer separation and identification of triacylglycerols containing vaccenic and oleic acids, and $\alpha$ - and $\gamma$-linolenic acids, in thermophilic cyanobacteria Mastigocladus laminosus and Tolypothrix sp. Phytochemistry 78: 147-155.

Schuyl PJW, de Joode T, Vasconcellos MA, Duchateau GSMJE. 1998. Silver-ion high-performance liquid chromatographyelectrospray mass spectrometry of triacylglycerols. $J$. Chromatogr. A 810: 53-61.

Semporé G, Bézard J. 1986. Qualitative and quantitative analysis of peanut oil triacylglycerols by RP-HPLC. J. Chromatogr. 366: 261-282.

Stołyhwo A, Colin H, Guiochon G. 1985. Analysis of Triglycerides in Oils and Fats by Liquid Chromatography with the Laser Light Scattering Detector Anal. Chem. 57: 1342-1354.

Tchapla A. Héron S, Lesellier E, Colin H. 1993. General view of molecular interaction mechanisms in reversed-phase liquid chromatography. J. Chromatogr. A 656: 81-112.

Ucciani U. 1995. Nouveau Dictionnaire des huiles végétales (Composition en acides gras). Paris : Technique et Documentation- Lavoisier.

Yang K, Zhao Z, Gross RW, Han X. 2011. Identification and Quantitation of Unsaturated Fatty Acid Isomers by Electrospray Ionization Tandem Mass Spectrometry : A Shotgun Lipidomics Approach. Anal. Chem. 83: 4243-4250.

Yang Q, Shi X, Gu Q, Zhao S, Shan Y, Xu G. 2012. On-line two dimensional liquid chromatography/mass spectrometry for the analysis of triacylglycerides in peanut oil and mouse tissue. $J$. Chromatogr. B 895-896: 48-55.

Yeboah SO, Mitei YC, Ngila JC, Wessjohann L, Schmidt J, 2011 Compositional and structural studies of the major and minor components in three cameroonian seed oils by GC-MS, ESI-FTICRMS and HPLC. J. Am. Oil Chem. Soc. 88: 1539-1549.

Zeitoun MAM, Neff WE, Selke E, Mounts TL. 1991. Analyses of vegetable oil triglyceride molecular species by reversed phase high perfomance liquid chromatography. J. Liq. Chrom. 14: 2685-2698.

Zeng Y.Z, Araugo P, Du Z.Y, Nguyen T.T, Frøyland, Grund B. 2010. Elucidation of triacylglycerols in cod liver oil by liquid chromatography electrospray tandem ion-trap mass spectrometry. Talanta 82: $1261-1270$

Cite this article as: Arnaud W. G. Tamba Sompila, Marie Geneviève Maloumbi, Jean Bleton, Alain Tchapla, Sylvie Héron. Identification des triacylglycérols en HPLC. Comment se passer du couplage HPLC-SM? Dans quel cas la chromatographie est-elle encore indispensable ? OCL 2014, 21(6) A601. 